\title{
Lessons of the month 4: Giant cell arteritis with normal inflammatory markers and isolated oculomotor nerve palsy
}

\author{
Authors: Benjamin Walters, ${ }^{A}$ Darko Lazic, ${ }^{B}$ Azeem Ahmed ${ }^{C}$ and Gabriel Yiin ${ }^{D}$
}

Giant cell arteritis (GCA) is an important condition to suspect and treat early, as failure to do so can result in anterior ischaemic optic neuropathy and subsequent permanent visual loss.

A 71-year-old woman presented to her local emergency department with a 1-week history of constant, moderatesevere global headache associated with intermittent periorbital pain. Two weeks later she developed sudden horizontal diplopia. Examination demonstrated right oculomotor nerve palsy. Her erythrocyte sedimentation rate (ESR) was $9 \mathrm{~mm} / \mathrm{hr}$. Repeat blood tests 1 month later showed an ESR of $67 \mathrm{~mm} / \mathrm{hr}$. Temporal artery biopsy was positive.

A review from a cohort of 764 patients with suspected GCA who underwent biopsy found the sensitivity of an elevated ESR and C-reactive protein was $84 \%$ and $86 \%$, respectively, but the specificity was only $30 \%$. Therefore, inflammatory markers should only act as a guide, and caution should be taken in their interpretation especially with respect to the time of sampling in the disease evolution.

Isolated oculomotor nerve palsy in association with GCA is rare. The first case series was described by Miller Fisher in 1959 who observed two patients presenting with diplopia, ptosis and ocular palsies. In anyone over the age of 50 who develops a new, refractory headache and cranial neuropathy, GCA should be the first consideration.

KEYWORDS: Giant cell arteritis, ocular palsy, normal inflammatory markers

DOI: 10.7861/clinmed.2019-0504

\section{Introduction}

Giant cell arteritis (GCA) is a medium to large sized vessel vasculitis that classically effects the extracranial vessels within the temporal region. In the UK it has an annual incidence of 20 per 100,000 people. 'GCA is an important condition to suspect and treat early, as failure to do so can result in anterior ischaemic optic neuropathy and subsequent

Authors: ${ }^{\text {A }}$ linical teaching fellow, Great Western Hospital, Swindon, UK; ${ }^{B}$ consultant histopathologist, Great Western Hospital, Swindon, UK; ${ }^{C}$ consultant rheumatologist, Great Western Hospital, Swindon, UK; ${ }^{\mathrm{D}}$ consultant neurologist, Great Western Hospital, Swindon, UK permanent visual loss. Due to its inflammatory pathophysiology, the American College of Rheumatology include an erythrocyte sedimentation rate (ESR) of $>50 \mathrm{~mm} / \mathrm{hr}$ as one of its five classification criteria. ${ }^{2}$ This case demonstrates that though unusual, at time of diagnosis, inflammatory markers can be normal and therefore a normal ESR or C-reactive protein (CRP) should not falsely reassure clinicians.

\section{Case presentation}

\section{A 71-year-old woman presented to her local emergency} department (ED) with a week history of a constant, moderatesevere global headache associated with intermittent periorbital pain. She re-presented to ED 2 weeks later as she woke up with sudden horizontal diplopia and later transferred to the stroke unit. She also had mild mid-facial pain but there was no pelvic/shoulder girdle pain, scalp tenderness or jaw claudication. Ophthalmological assessment determined a mild right oculomotor nerve palsy and fundoscopy revealed some extramacular drusen. She had an ESR of $9 \mathrm{~mm} / \mathrm{hr}$, a CRP of $4.7 \mathrm{mg} / \mathrm{L}$ and normal full blood count. Computed tomography angiography excluded a posterior communicating artery aneurysm. Magnetic resonance imaging of the head was normal and muscle specific kinase/anti-acetylcholine receptor antibodies were negative.

At this stage, the differential diagnosis was that of ophthalmoplegic migraine or microvascular oculomotor nerve palsy. She was discharged home with stroke secondary prevention medications and amitriptyline. A follow-up appointment was arranged. Her headache did not significantly improve with amitriptyline and she visited a private neurologist 4 weeks after discharge. Repeat blood tests revealed an ESR of $67 \mathrm{~mm} / \mathrm{hr}$ and she was referred back to neurology/rheumatology locally and commenced on $30 \mathrm{mg} /$ day of prednisolone. A full autoimmune screen was negative. Her headache and diplopia settled within 3 days and she was placed on a reducing dose of prednisolone with omeprazole, alendronic acid and Adcal-D3. A temporal artery biopsy (TAB) confirmed the diagnosis 3 months following her initial presentation while she was on prednisolone (Fig 1). Despite the initial delay in diagnosis and treatment, she has had no lasting effects to her vision and no longer suffers from diplopia.

\section{Histology}

Sections reveal an arterial wall with focally disturbed architecture, narrowed lumen and an inflammatory cell infiltrate; the 


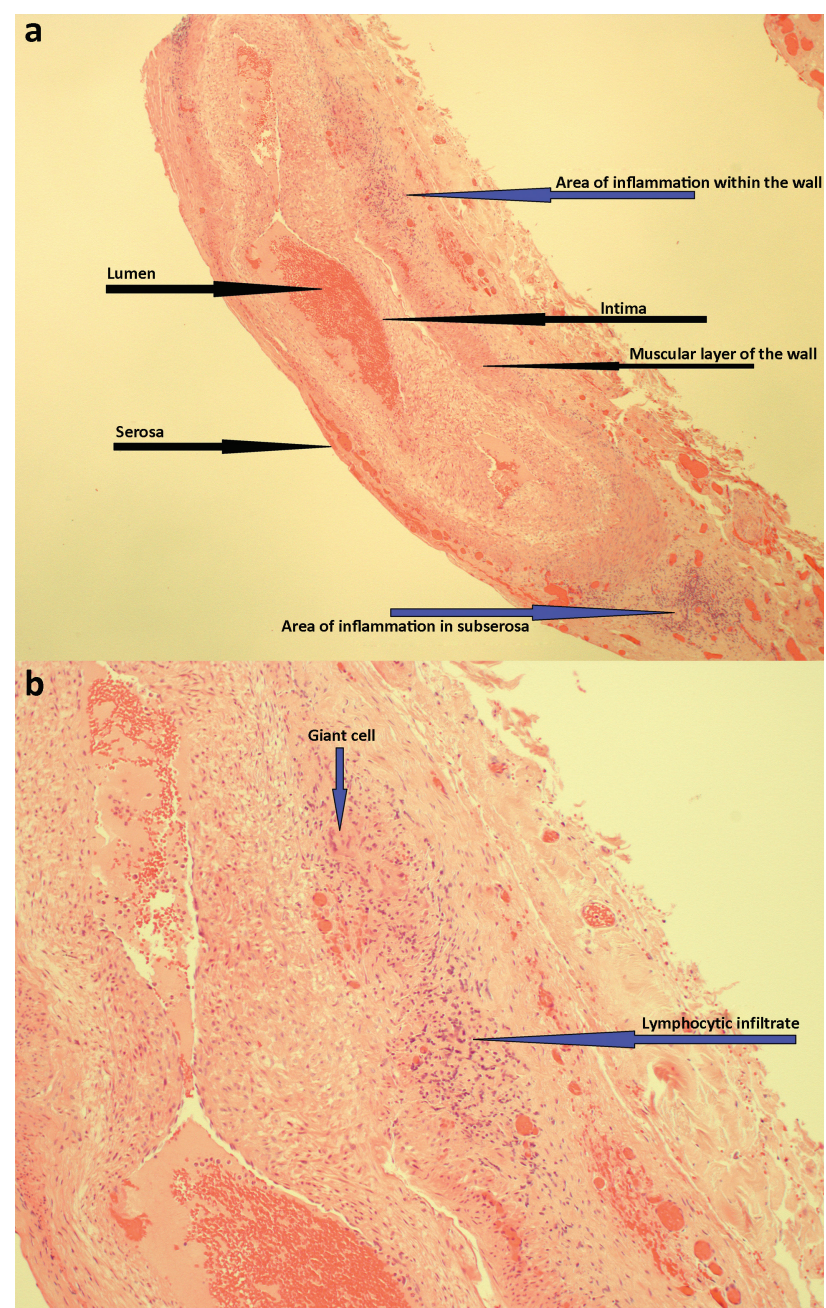

Fig 1. Temporal artery biopsy histology showing giant cell arteritis. a) At 40x magnification. b) At 100x magnification.

inflammation is focal and spreads from the subserosa into the wall; occasional multinucleated giant cells are noted (Fig 1).

\section{Discussion}

In 2002, Gerald et al published a meta-analysis to ascertain the accuracy of the history, examination and ESR in the diagnosis of GCA. ${ }^{3}$ From 21 core studies and over 2,000 patients, ESR was deemed most useful as a marker for the probability of a positive TAB. Among patients clinically suspected of disease, those with an ESR greater than $100 \mathrm{~mm} / \mathrm{hr}$ have a modestly increased likelihood of biopsy-proven GCA (likelihood ratio 1.9). Conversely, only $4 \%$ of patients with a positive biopsy had a normal ESR. As such, it concluded that a normal ESR makes GCA unlikely. However, another review found that in a cohort of 764 patients with suspected GCA who underwent biopsy, even though the sensitivity of an elevated ESR and CRP was $84 \%$ and $86 \%$, respectively, the specificity of ESR/CRP was only $30 \%$. $^{4}$ Therefore, inflammatory markers should only act as a guide and caution should be taken in their interpretation especially with respect to the time of sampling in the disease evolution. If a patient presents with supportive clinical features but normal inflammatory markers, it is reasonable to treat as GCA until TAB result is available. Please see supplementary material $\mathrm{S} 1$ which summarises five other published case reports of GCA with normal inflammatory markers. ${ }^{5-8}$

The hypothesis as to why patients can have an initial normal ESR/CRP at diagnosis but that increase at a later date, as it was in this case and the case reported by Yu-Wai-Man et al, was touched upon by Salvarani et al. ${ }^{8,9}$ It was believed that a genetically determined inhibition or poor initiation in cytokine and complement cascades may well be an underlying cause and contributing factor.

As many as $15 \%$ of GCA can have a negative TAB. ${ }^{4}$ An old study found that even though $82 \%$ of patients had a positive biopsy before steroid treatment, this reduced to $60 \%$ after 7 days of steroid treatment and $10 \%$ thereafter. ${ }^{10}$ However, our case has demonstrated that there is exception to this rule. This is supported by a recent study that demonstrated the persistence of the characteristic granulomatous inflammation in $50 \%$ and $25 \%$ of repeat biopsies after treatment at 9 and 12 months, respectively. ${ }^{11}$

Isolated oculomotor nerve palsy in association with GCA is rare. The first case series was described by Miller Fisher in 1959 who observed two patients presenting with diplopia, ptosis and ocular palsies. ${ }^{12}$ As headache was not a predominant feature, GCA was only suspected and subsequently confirmed on biopsy after they had marked visual impairment. Interestingly, steroids resulted in rapid and complete resolution of oculomotor palsies but had no effect on visual loss.

\section{Conclusion}

We present a rare manifestation of GCA with normal inflammatory markers and isolated oculomotor nerve palsy. In anyone over the age of 50 who develops a new, refractory headache and cranial neuropathy, GCA should be the first consideration. Inflammatory markers should be repeated if normal at initial testing and temporal artery biopsy considered early. Importantly, there should be no delay in starting steroid treatment so as to avoid progression into permanent visual loss.

\section{Supplementary material}

Additional supplementary material may be found in the online version of this article at www.rcpjournals.org/content/clinmedicine: S1 - Summary of other case reports.

\section{References}

1 National Institute for Health and Care Excellence. Giant cell arteritis. NICE, 2014. https://cks.nice.org.uk/giant-cellarteritis\#!topicSummary [Accessed 29 October 2019].

2 Hunder GG, Bloch DA, Michel BA et al. The American College of Rheumatology 1990 criteria for the classification of giant cell arteritis. Arthritis Rheum 1990;33:1122-8.

3 Smetana G, Shmerling RH. Does this patient have temporal arteritis? JAMA 2002;287:92.

4 Weyand C, Goronzy J. Giant cell arteritis and polymyalgia rheumatica. N Engl J Med 2014;371:50-5.

5 Espinoza LR, Espinoza CG. Temporal arteritis with normal ESR. Arch Intern Med 1980;140:281-2.

6 Kansu T, Corbett J, Savino P, Schatz N. Giant cell arteritis with normal sedimentation rate. Arch Neurol 1977;34:624-5. 
7 Poole T, Graham E, Lucas S. Giant cell arteritis with a normal ESR and CRP. Eye 2003;17:92-3.

8 Yu-Wai-Man P, Dayan M. Giant cell arteritis with normal inflammatory markers. Acta Ophthalmol Scand 2006;85:460.

9 Salvarani C, Hunder G. Giant cell arteritis with low erythrocyte sedimentation rate: Frequency of occurrence in a population-based study. Arthritis Rheum 2001;45:140-5.

10 Allison M, Gallagher P. Temporal artery biopsy and corticosteroid treatment. Ann Rheum Dis 1984:43:416-7.
11 Maleszewski J], Younge BR, Fritzlen JT et al. Clinical and pathological evolution of giant cell arteritis: a prospective study of follow-up temporal artery biopsies in 40 treated patients. Mol Pathol 2017;30:788-96.

12 Fisher M. Ocular palsy in temporal arteritis. Minneapolis: Minnesota Medicine Association, 1959:1258-68.

Address for correspondence: Dr Benjamin Walters, Great Western Hospital, Marlborough Road, Swindon SN3 6BB, UK. Email: benjamin.walters@nhs.net

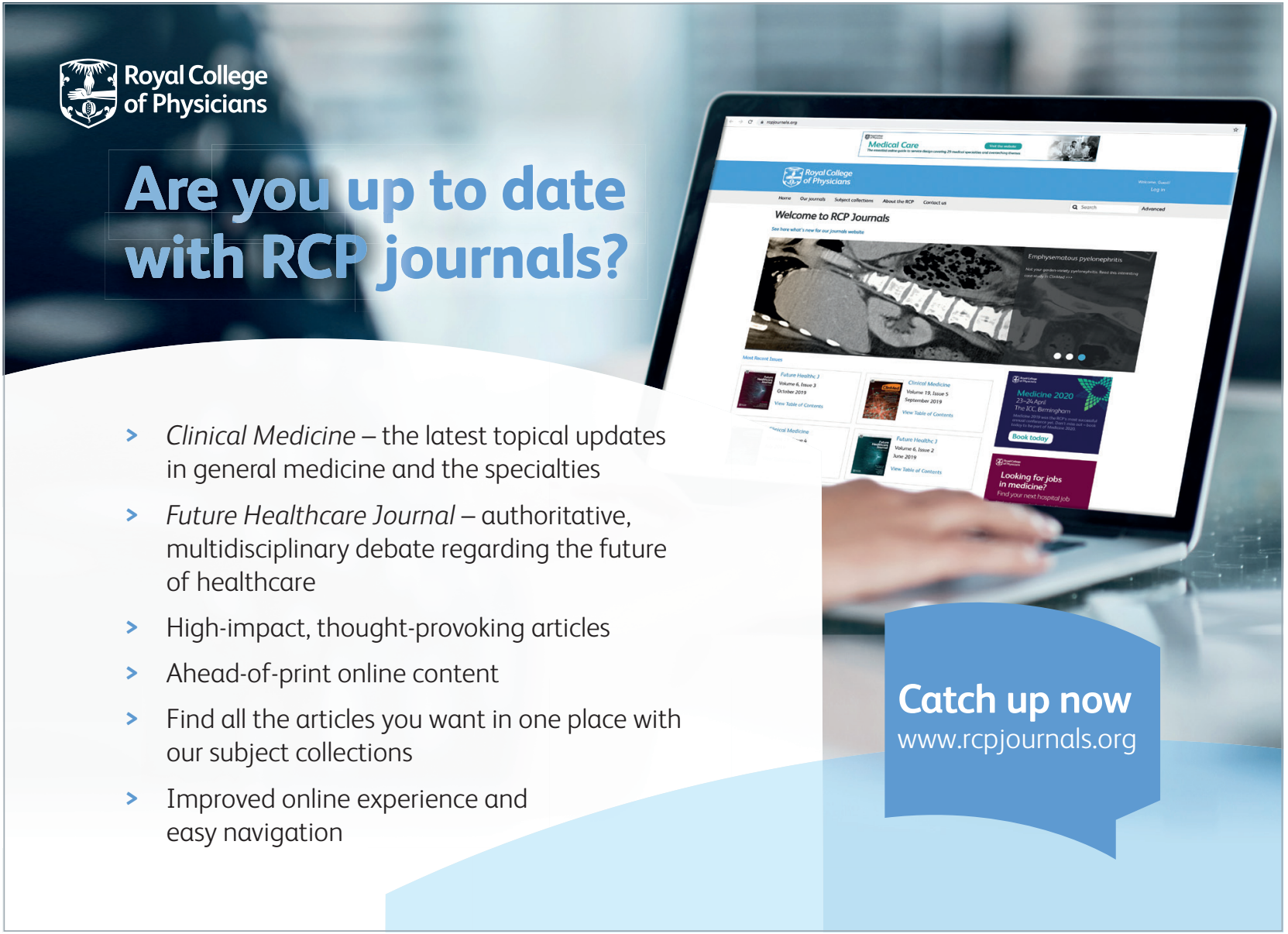

